\title{
Erratum: Influence of network topology on sound propagation in granular materials [Phys. Rev. E 86, 041306 (2012)]
}

\author{
Danielle S. Bassett, Eli T. Owens, Karen E. Daniels, and Mason A. Porter \\ (Received 10 August 2015; published 29 September 2015) \\ DOI: 10.1103/PhysRevE.92.039905 \\ PACS number(s): 81.05.Rm, 81.70.Cv, 89.75.Fb, 89.75.Hc, 99.10.Cd
}

Computations. In our paper, some of our computations were for quantities that were similar to those that we reported in our formulas, but they were not quite the same. Below we provide the corrected formulas.

For weighted networks, we calculated a local version of a clustering coefficient. [See Eq. (A20) on p. 12 of our paper.] Let $k_{i}$ be the (unweighted) degree of node $i$, and let $\hat{\mathbf{W}}$ denote the weighted adjacency matrix. The contribution $N_{\mathrm{cyc}_{3}}(i)$ from length-3 cycles that include node $i$ is equal to the $(i, i)$ th element of the matrix $\hat{\mathbf{W}}$, where

$$
\begin{aligned}
\hat{\mathbf{W}} & =\mathbf{Y}^{3}, \\
Y_{i j} & =W_{i j}^{1 / 3}, \quad i, j \in\{1, \ldots, N\} .
\end{aligned}
$$

We then calculated a local weighted clustering coefficient,

$$
C_{w}(i)=\frac{N_{\mathrm{cyc}_{3}}(i)}{k_{i}\left(k_{i}-1\right)},
$$

for all nodes $i$ that are part of at least one 3-cycle. For any node $i$ that is not part of a 3-cycle, we set $C_{w}(i)=0$. The code (CLUSTERING_COEF_WU.M) that we used for this computation comes from the Brain Connectivity Toolbox [1]. To subsequently calculate a global weighted clustering coefficient, we computed $C_{w}=\frac{1}{N} \sum_{i} C_{w}(i)$.

For weighted networks, we also calculated a second type of global clustering coefficient. [See Eq. (A24) on p. 13 of our paper.] We computed $N_{\mathrm{cyc}_{3}}(i)$ as above, and we then calculated a global weighted transitivity using the formula

$$
T=\frac{\sum_{i} N_{\mathrm{cyc}_{3}}(i)}{\sum_{i} k_{i}\left(k_{i}-1\right)}
$$

for all nodes $i$. The code (TRANSITIVITY_WU.M) that we used for this computation comes from the Brain Connectivity Toolbox [1].

Typographical errors. Our formula [see Eq. (A2) on p. 9 of the paper] for the geodesic node betweenness centrality of node $i$ in an unweighted network should read

$$
B_{i}=\sum_{j, m \in \mathcal{G}} \frac{\psi_{j, m}(i)}{\psi_{j, m}}
$$

where all three nodes $(j, m$, and $i)$ must be different from each other, $\psi_{j, m}$ is the number of geodesic paths between nodes $j$ and $m$, and $\psi_{j, m}(i)$ is the number of geodesic paths between $j$ and $m$ that traverse node $i$. Our computations of node betweenness for unweighted networks in the paper used Eq. (4), but the equation in our paper makes it look like we were summing over $i$. That is not the case, as there is a value of geodesic node betweenness for each node $i$. The same change corrects our equation [see Eq. (A21) on p. 12 of the paper] for geodesic node betweenness centrality in a weighted network, and again all computations in our paper used the correct equation. The code (BETWEENNESS_BIN.M and BETWEENNESS_WEI.M) that we used for this computation comes from the Brain Connectivity Toolbox [1].

Our formula [see Eq. (A3) on p. 9 of the paper] for the geodesic edge betweenness centrality of edge $(j, m)$ that connects nodes $j$ and $m$ in an unweighted network should read

$$
B_{e}(j, m)=\sum_{i, k} \frac{\psi_{i, k}(j, m)}{\psi_{j, m}},
$$

where $\psi_{j, m}$ is again the number of geodesic paths between nodes $j$ and $m$, and $\psi_{i, k}(j, m)$ is the number of geodesic paths between $i$ and $k$ that traverse the edge that connects nodes $j$ and $m$. That is, we used the same type of normalization as in our computation of geodesic node betweenness centrality. The same comment about normalization applies to our equation [see Eq. (A22) on p. 12 of the paper] for geodesic edge betweenness centrality in a weighted network. The code (EDGE_BETWEENNESS_BIN.M and EDGE_BETWEENNESS_WEI.M) that we used for this computation comes from the Brain Connectivity Toolbox [1].

In our equation [see Eq. (A10) on p. 10 of the paper] for the local efficiency of node $i$ in an unweighted network, we neglected to state explicitly that $N_{G_{i}}$ represents the number of nodes in the subgraph $G_{i}$. 
As we noted earlier in this Erratum, our formula [see Eq. (A24) on p. 13] for the global transitivity in a weighted network should read

$$
T=\frac{\sum_{i} N_{\mathrm{cyc}_{3}}(i)}{\sum_{i} k_{i}\left(k_{i}-1\right)}
$$

for all nodes $i$. The code (TRANSITIVITY_WU.M) that we used for this computation comes from the Brain Connectivity Toolbox [1]. The difference from the formula [see Eq. (A24) on p. 13] in our paper is that the denominator should be $k_{i}\left(k_{i}-1\right)$ rather than $k_{i}\left(k_{i}+1\right)$. All of the computations in our paper used the correct factor [i.e., $k_{i}\left(k_{i}-1\right)$ ] in the denominator.

Finally, as a trivial correction, the phrase "edge-connecting node $i$ and node $j$ " on p. 3 should instead read "edge that connects nodes $i$ and $j$ " (i.e., there should not be any hyphen).

Acknowledgments. We thank J. Setford for his help in uncovering several of the above issues.

[1] M. Rubinov and O. Sporns, Brain Connectivity Toolbox (2010), https://sites.google.com/site/bctnet/. 